\title{
Caractéristiques des patients admis au service d'accueil des urgences du Centre Hospitalo-Universitaire du Point G, Bamako, Mali
}

\section{Epidemiological characteristics of patients admitted to the emergency's reception of the University Hospital of Point G, Bamako, Mali.}

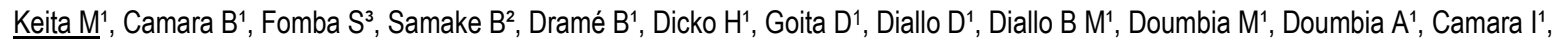
Coulibaly I', Doumbia $\mathrm{D}^{1}$, Coulibaly $\mathrm{Y}^{1}$

${ }^{1}$ Service Anesthésie - Réanimation et des Urgences centre hospitalier universitaire (CHU) du Point G

${ }^{2}$ Service Anesthésie - Réanimation et des Urgences CHU Gabriel TOURE

${ }^{3}$ Programme national de lutte contre le paludisme (PNLP), Mali

\section{Résumé :}

L'objectif de cette étude était de décrire et d'analyser les caractéristiques épidémiologiques des patients admis pour urgences médicales au service d'accueil des urgences du Centre Hospitalo- Universitaire du Point G. II s'est agit d'une étude prospective, descriptive et analytique de suivi de tous les patients admis de Juillet 2012 à Juin 2013. Pendant la période d'étude, 2254 dossiers ont été colligés. Le nombre médian d'admission journalière par mois était de 26,83 $\pm 2,95$ patients. L'âge moyen était de $49 \pm 20$ ans. Mille sept cent soixante dix sept patients avaient fréquenté une à trois structure (s) de soins avant leur admission dans ladite structure, la durée d'évolution de leur maladie était $>7$ jours dans $52,9 \%$ des cas, $59,5 \%$ avaient été référés et $25 \%$ avait été amenés par ambulance. Les plaintes les plus nombreuses à l'admission étaient l'asthénie physique/Anorexie, les fièvres/frissons et les vertiges. Mille cinq cent quatre vingt quinze patients $(71 \%)$ avaient été "jugés graves". La pathologie diagnostiquée était d'origine non infectieuse dans $40 \%$ des cas, infectieuse dans $39 \%$ cas et frustre dans $21 \%$ des cas. La pathologie non infectieuse était le motif de recours au SAU chez les patients "jugés graves"dans $80 \%$ des cas. La durée moyenne d'hospitalisation était de $2,61 \pm 5,30$ jours.

La mortalité $(18,5 \%)$ était significativement plus élevée chez les patients"jugés graves"

$(p=0,001)$.

Les résultats de notre étude incitent à entreprendre des actions de communication avec la population pour promouvoir le bon usage du service des urgences.

Mots clés: Caractéristiques patients, Service accueil, Urgence, Point G

\footnotetext{
Abstract:

This research aimed to describe the epidemiological characteristics and to analyze predictive factors of clinical status severity for patients admitted to the emergency service of University Hospital of Point G.

We used an prospective, descriptive and analytical study and followed up all patients admitted to the emergency service of University Hospital of Point G from July 2012 to June 2013.Data about their clinical status, diagnostic and evolution have been described. The severity of the clinical status of patients was determined by using the Clinical Classification of Patients in emergency.
}

During the study period, 2254 files were collected. The median number of daily admissions per month was 26.83 \pm 2.95 patients. The mean age was $49 \pm 20$ years. One thousand seven hundred seventy seven patients used one to three health structure(s) prior to their admission to the University Hospital. The disease's evolution was $>7$ days in $52.9 \%$ of cases, $59.5 \%$ were referred and $25 \%$ had been transported by ambulance. The frequently complaints named at admission were physical asthenia / anorexia, fever / shiver and dizziness. One thousand five hundred ninety five patients $(71 \%)$ were "considered serious." The diseases diagnosed were noninfectious in $40 \%$ of cases, infectious in 39\% cases and unclear in 21 $\%$ of cases. Noninfectious disease was the reason for the University Hospital use for patients «judged sever» in 80 $\%$ of cases. The average hospital stay was $2.61 \pm 5.30$ days. Mortality rate $(18.5 \%)$ was significantly higher in patients «judged sever» $(p=0.001)$. These results must push the health authorities to take communication actions toward the public for the promotion of best use of emergencies services.

Keywords: Patient's characteristics, Entrance Service, mergency, Point $\mathrm{G}$.

\section{Introduction}

La médecine d'urgence est un centre d'intérêt majeur en médecine curative aussi bien dans les pays développés que dans les pays en voie de développement.

Les services d'urgence (SU) du monde entier constituent un maillon vital entre la prise en charge pré hospitalière et l'hospitalisation des consultants [1,2].

Au cours des dernières décennies, les SU des hôpitaux n'ont cessé de voir leur activité augmenter [3]. Cette hausse constante de la fréquentation des services d'urgence est un phénomène commun à tous les pays qui en possèdent [2,4].

Au Canada en 2001, on enregistrait une augmentation du nombre de passages annuels aux urgences de $5 \%$ en 3 ans [5], alors qu'aux Etats-Unis, une croissance de $14 \%$ a été enregistrée entre 1992 et 1999 [6].

Par ailleurs, plusieurs pays de la Région africaine sont concernés par cette hausse du recours aux SU. En Tunisie, 1058 recours ont été enregistrés au SAU en 2009 [7], au Sénégal en 2008, 3115 patients avaient été consultés au service des urgences [8]. 
En zone tropicale, les urgences surviennent presque toujours dans un contexte socio - économique défavorable et dans un sous équipement médical important [9] malgré, la population y a recourt de plus en plus fréquemment.

Dans les pays développés, plusieurs évaluations ont été faites pour en ajuster le fonctionnement $[10,11]$.

Au Mali, l'activité et le profil des patients fréquentant le service d'accueil des urgences (SAU) ont été peu étudiés, alors que l'organisation rationnelle et l'adaptation

\section{Patients et méthodes}

II s'agissait d'une étude prospective, descriptive et de suivi de tous les patients admis au service d'accueil des urgences médicales du CHU du point G, Bamako, Mali de juillet 2012 à Juin 2013.Etaient inclus tous les patients quel que soit l'âge, la pathologie, admis au service d'accueil des urgences pour une urgence médicale, qui ont délibérément consentis (ou l'accompagnant a consenti) à participer à l'étude. Lesparamètres étudiés étaient: les caractéristiques sociodémographiques, l'histoire de la maladie, les modalités et l'heure d'admission, le motif de consultation, les signes cliniques, le diagnostic retenu et l'évolution. Pour l'itinéraire, était considéré comme référé médical lorsque l'arrivée du patient au SAU faisait suite à une décision ou orientation (verbale ou écrite) d'un personnel soignant, les autres patients étaient considérés comme auto référés. Ont été considérés comme médicalisés, les transports effectués par les ambulanciers. L'automédication était définie par l'utilisation des médicaments par le patient sans prescription médicale[12].

Les diagnostics ont été classés en groupes nosologiques selon la classification internationale des maladies (ICD-9 - CM).Les pathologies diagnostiquées étaient regroupées en origine infectieuse ou non infectieuse. En l'absence d'une orientation diagnostique claire, l'origine « imprécise » a été rapportée et ces cas ont été classés comme d'origine frustre. La gravité de l'état clinique des patients a été déterminée par la classification clinique des malades des urgences (CCMU) modifiée en sept classes $[13,14]$. Les patients des classes, 1 et 2 étaient "jugés stables" et ceux des classes 3,4 et 5 étaient "jugés graves", les patients de la classe D étaient ceux dont le décès était constaté à l'arrivée. [15]. La classe $P$ n'a pas été prise en compte dans l'étude.

Les données étaient recueillies sur des fiches d'enquête individuelles, les jours ouvrables (lundi à vendredi, excepté les jours fériés) versus les jours non ouvrables (samedi et dimanche) et fériés et les heures ouvrables (07h00 à 15h59) versus les heures non ouvrables (16h00 à 06h59). Les jours de consultation ont été regroupés en " début de semaine » (lundi et mardi), " en milieu de semaine » (mercredi, jeudi et vendredi) et « en fin de semaine » (samedi et dimanche).Une enquête préliminaire avait été réalisée pendant deux semaines du 1 au 15 Juillet 2012 en vue de vérifier la faisabilité du protocole.L'accord des patients a été demandé avant chaque inclusion. Lorsque le patient était dans l'incapacité de donner son accord, l'autorisation de l'accompagnant a été requise. Le refus ou l'adhésion plus efficiente de l'offre des soins requièrent des informations sur la nature de la demande (nombre de patients admis, types de pathologies rencontrées, coût des soins y afférant). II nous a donc semblé intéressant de mener une étude épidémiologique descriptive afin de determinerles caractéristiques épidémiologiques des patients admis pour urgences médicales au service d'accueil des urgences du CHU du Point G.

d'un patient n'avait aucune influence sur son traitement tout au long de son séjour.

La saisie et l'analyse statistique étaient réalisées avec les logiciels EPI data et SPSS version 17.0. Le Seuil de signification statistique était fixé à 0,05 .

\section{Résultats}

L'état comateux des patients, l'état psychologique des accompagnants et la culture chez les maliens de ne pas archiver les dossiers médicaux étaient les limites de notre étude.

Nous avons colligés 2254 sur 3000 dossiers de patients admis au service d'accueil des urgences du CHU du Point $G$ pour une urgence médicale. Le nombre de patients ayant refusé de participer à l'enquête était de 746 soit $25 \%$. Le nombre médian d'admission journalière par mois était de $26,83 \pm 2,95$ patients et le nombre médian d'admission par semaine était de 15,44 \pm 6,94 patients. Les admissions étaient plus nombreuses en milieu de semaine $(40,3 \%)$ contre $31,5 \%$ en début de semaine et $28,2 \%$ en fin de semaine. La distribution mensuelle des admissions est illustrée par la [Figure1]. Quarante deux patients, soit $2 \%$ des admissions venaient des pays limitrophes, il s'agit de la Guinée Conakry $(76 \%)$, de la Mauritanie (14\%) et de la côte d'ivoire (10\%). L'âge moyen était de $49 \pm 20$ ans avec des extrêmes allant de 14 à 99 ans. La répartition selon le sexe a montré un sexe ratio de 1,4. Mille sept cent soixante dix sept patients soit $84,7 \%$ avaient fréquenté une à trois structure (s) de santé avant leur admission au SAU. La durée d'évolution de la maladie était $>7$ jours dans $52,9 \%$ des cas, et $59,5 \%$ des cas avaient été référés [Tableau1]. Pour ce qui concerne les moyens de transport utilisés par le patient pour se rendre à l'hôpital, $25 \%$ ont été amenés par ambulance contre $41 \%$ et $30 \%$ respectivement par transport en commun et voiture privée. Les admissions étaient plus nombreuses les jours ouvrables et aux heures non ouvrables $(62,4 \%$, soit 258 patients). Les symptomatologies les plus fréquentes des patients à l'admission étaient l'asthénie physique ou l'anorexie chez $69,52 \%$ des patients (1567cas), les fièvres ou frissons chez $58,43 \%$ des patients (1317 cas), les vertiges chez $55,64 \%$ des patients (1254 cas) et les nausées ou vomissements/diarrhées chez $50,18 \%$ des patients (1131cas).

L'étude des circonstances de la consultation a montré que $11,5 \%$ avaient consulté durant les heures ouvrables, $71,8 \%$ un jour ouvrable (Lundi à Vendredi), $28,2 \%$ un jour non ouvrable (Samedi et Dimanche) et seulement 3,4\% un jour férié.

Mille cinq cent quatre vingt quinze patients $(71 \%)$ ont été "jugés graves", 504 patients (22\%) étaient"jugés stables" 
[Figure 2]. La pathologie diagnostiquée était d'origine infectieuse dans 39\% cas, non infectieuse dans $40 \%$ des cas et imprécise dans $21 \%$ des cas. La pathologie non infectieuse était le motif de recours au SAU chez les patients "jugés graves"dans $80 \%$ des cas et dans $20 \%$ des cas chez les patients "jugés stables", tandis que le motif de recours au SAU pour pathologie infectieuse était de $74,2 \%$ pour les patients "jugés graves" et de $25,8 \%$ pour les patients "jugés stables". Les pathologies infectieuses étaient virales $(45,1 \%)$, parasitaires $(35,7 \%)$ et bactériennes $(19,2 \%)$, [figure 2]. Les principales pathologies non infectieuses étaient neurologiques $(28,3 \%)$, digestives $(22,3 \%)$, rénales $(17,8 \%)$ et endocrinologiques (10,6\%) [Figure3]. La distribution des pathologies cardiovasculaires était l'hypertension artérielle $(67 \%)$, l'accident vasculaire cérébral $(22 \%)$ et l'insuffisance cardiaque (11\%). Les complications de l'hypertension artérielle étaient l'accident vasculaire cérébral (59\%), l'insuffisance cardiaque $(24 \%)$ et l'insuffisance rénale (17\%). Soixante dix neuf pour cent des patients ont été hospitalisés pendant une durée moyenne de 2,61 $\pm 5,30$ jours. L'analyse de la gravité de l'état clinique du patient est illustrée dans le [Tableau2] La létalité était de $23 \%$ chez les patients dont l'origine de la pathologie était non infectieuse contre $47 \%$ pour les patients chez lesquels l'origine de la pathologie était infectieuse. Rapportée aux patients "jugés graves" ( $n=$ $350)$, la létalité atteint $22 \%(p=0,001)$ [Figure 4]. Les causes probables de décès étaient liées dans $97 \%$ des cas à la gravité de l'état clinique des patients.

\section{Discussion}

Les urgences médicales sont fréquentes dans notre contexte de travail comme en atteste le nombre élevé d'admissions (2254) en 12 mois. Cette fréquence est essentiellement liée au fait que le District de Bamako, qui représente $267 / 1241300 \mathrm{~km}^{2}$ soit $0,02 \%$ du territoire national, concentre 2094000/ 16723269 habitants soit12,52\% de la population malienne avec un taux d'accroissement de 2,2\% [16]. K Sall et al [9] à Dakar, retrouvent également que l'urgence constitue le mode d'admission le plus habituel en milieu tropical.

Notre étude permet de décrire la population qui a fréquenté le SAU du CHU du Point G pendant la période d'étude. II apparaît que $57,7 \%$ d'hommes a consulté le SAU contre $42,3 \%$ de femmes. La prédominance du sexe masculin s'explique par le fait que les urgences obstétricales ont été exclues de ce travail. Roger - Leroi. $V$ et al [17], Mbutiwi IN [23] et al et K Sall et al [9] ont également montré une plus forte fréquentation masculine alors que les études menées dans d'autres pays n'avaient pas noté de différence selon le sexe $[8,19]$.

Le nombre médian d'admission journalière par mois était de $26,83 \pm 2,95$, notre résultat est similaire a celui trouvé par TRAORE et al au Burkina FASO [19].

Dans notre population d'étude, la moyenne d'âge était de $49 \pm 20$ ans. Les deux sexes étaient inéquitablement représentés dans notre échantillon $(57,7 \%$ d'hommes contre 42,3\% de femme). En 2010, une étude réalisée au service d'urgence du centre hospitalier provincial de Nador au Maroc avait retrouvé une moyenne d'âge de
$36,7 \pm 15,7$ ans. Dans cette étude, on notait une légère prédominance masculine [20]. Plus des $2 / 3$ des admissions au SAU viennent de Bamako et de sa périphérie, ce qui correspond aux résultats d'autres études $[9,17,21]$ selon lesquelles la proximité du service est un critère de choix pour les patients. Les catégories socioprofessionnelles les plus représentées étaient les femmes au foyer, les cultivateurs, les commerçants et les employés privés. Les patients n'ayant effectué aucune étude $(59,2 \%)$ avaient les plus fréquentés les services de santé. Cette représentation des catégories professionnelles est retrouvée dans les études de Roger et al [17] et de Berraho et al [20].Le profil des patients était caractérisé par l'importance des références médicales $(59,5 \%)$. Elles étaient le fait des admissions de Bamako et périphérie (902 admissions) elles étaient 2fois plus importantes que chez les autres admissions. Mille sept cent soixante dix sept patients, soit $63,5 \%$ avaient fréquenté une à trois structure (s) de santé avant leur admission au SAU, la durée d'évolution de leur maladie était $>7$ jours $(52,9 \%)$ et $25 \%$ ont été amenés par ambulance. Le moyen de transport (Transport en commun, voiture privée, ambulance), le mode de référence étaient associés à la gravité du patient, par contre l'itinéraire n'était pas associé à la gravité. Abdoulaye et al [19], dans son étude, trouve l'itinéraire associé à la gravité. Nous avons retrouvé une relation significative $(p=0,001)$ entre l'état clinique du patient « jugé grave » et la tranche d'âge, le niveau d'étude, la durée d'évolution de la maladie et le mode de référence. La répartition temporelle des patients était inégale, le flux ayant été plus important en milieu de semaine avec 17 patients qu'en début et fin de semaine avec 14 et 12 patients. Abdoulaye et al [19] trouve un flux de patients plus important en début de semaine qu'en fin de semaine. Le principal moyen de transport utilisé était de loin « un transport en commun (41\%), le transport par ambulance ne représentait que $25 \%$. Notre résultat est similaire à celui d'une étude Marocaine [7] qui a observé un faible taux d'utilisation des ambulances comme moyen de transport pour accéder aux services des urgences (2,4\%).L'étude des circonstances de la consultation a montré que $11,5 \%$ des patients avait consulté durant les heures ouvrables, $71,8 \%$ un jour ouvrable (Lundi à Vendredi), 28,2\% un jour non ouvrable (Samedi et Dimanche) et seulement 3,4\% un jour férié. Dans l'étude de. Ben Gobrane [7] et al sur les Motifs de recours aux services d'urgence des principaux hôpitaux du Grand Tunis, 34,5\% des patients avaient consulté durant les heures ouvrables. Berraho et al [20] retrouvent $70,5 \%$ et $19,3 \%$ de patients qui ont consulté pour respectivement un jour ouvrable et un jour non ouvrable. Notre étude retrouve $3,4 \%$ patients qui ont consulté un jour férié, Berraho et al [20] retrouvent $10,2 \%$.

Les pathologies non infectieuses (72\%) occupaient le devant des tableaux cliniques documentés au niveau du SAU du CHU du Point G.A Dakar, une étude réalisée au SAU du CHN de Pikine trouvait une prédominance des pathologies infectieuses $(55,24 \%)$ [8].

Les accidents vasculaires cérébraux (AVC) et l'hypertension artérielle (HTA) représentaient plus du tiers des pathologies non infectieuses neurologiques 
(21\%). La non disponibilité de la Tomodensitométrie (Scanner) en urgence est un handicap pour le diagnostic de certitude des AVC. Seuls 15 cas ont été confirmés. Les patients qui en bénéficiaient, étaient transférés par ambulance ou en voiture privée vers des centres hospitaliers disposant d'un scanner. Touré [8] et al à Dakar avaient trouvé un résultat similaire.

L'insuffisance cardiaque (24\%) et l'insuffisance rénale (17\%) représentaient les complications de l'hypertension artérielle.

La proportion des patients admis au SAU du CHU du Point $\mathrm{G}$ dans un état grave était de $76 \%$ Ben Gobrane et al [7] au Maroc avait retrouvé dans son étude $6,3 \%$ de proportion de patient se trouvant dans un état clinique grave. Cette différence s'explique dans notre contexte par le manque de tiers payant, obligeant le patient à ne consultéer que lorsque la maladie dont il souffre s'aggrave. Nous avons trouvé une association significative entre la tranche d'âge, le niveau d'étude, la durée d'évolution de la maladie, le mode de référence et la gravité de l'état clinique du patient $(p<0,001)$. Cependant, Berraho et al [20] rapportent dans leurs travaux que le caractère auto -référé des patients à l'admission au SAU n'était pas associé à la gravité de l'état clinique.

La majeure partie de patients (79\%) avaient été hospitalisée pour une durée moyenne d'hospitalisation de 2,61士5,30 jours. Mbutiwi IN et al[23] avaient retrouvé aux CUK de Kinshasa en RDC durée moyenne d'hospitalisation de 10 jours. Nous avons enregistré 19\% des admissions de patients qui retournaient chez eux après la consultation. $\mathrm{Ce}$ qui témoigne que le SAU ressemblait à un service de médecine générale, où le patient venait de lui-même. Notre taux est loin de celui retrouvé à l'hôpital principal de Dakar (HPD) en 2007 - 2008 [22] qui était de 80\%. Ce phénomène constitue la base de plusieurs problèmes dans nos services d'urgences actuels. Ce qui engendre un manque de place pour certaines "vraies urgences" qui font le tour des hôpitaux de Bamako pour être prise en charge. En occident, c'est une problématique tout aussi présente, Gentile et al [3] rapportaient que "la surpopulation des services d'urgence est en partie due à l'utilisation des services d'urgence par les patients non urgents ... »

L'automédication, le jour d'admission, l'heure d'admission et l'origine de la pathologie n'étaient pas associés à la gravité de l'état clinique. La mortalité au SAU du CHU du Point G était de $23 \%$, elle était plus élevée que celle notée aux CUK de Kinshasa en RDC (12,3\%) [23], au SAU du centre hospitalier national de Pikine $(2,2 \%)$ [8] et de l'hôpital principal de Dakar $(0,7 \%)$ [22]. La mortalité était plus importante parmi les patients chez qui l'origine de la pathologie était infectieuse (47\%).

\section{Conclusion}

La fréquentation du SAU du CHU du Point G est caractérisée par le recours massif des patients« jugés graves » $(74,23 \%)$ et qui y accèdent suite à une référence médicale $(59,5 \%)$.

Par leur fréquence, gravité et létalité élevées, les pathologies non infectieuses, posent un problème majeur de santé publique dans des urgences médicales SAU du CHU du Point G.
Des programmes de lutte contre ces pathologies non infectieuses devraient être redynamisés, en mettant l'information et l'éducation de la population au centre de toute action préventive.

Une analyse plus approfondie du fonctionnement du SAU permettrait d'identifier les

défaillances et de cibler les ajustements utiles afin de garantir une gestion efficace et efficiente des urgences médicales.

\section{References}

1. Rehmani R, Norain A. Trends in emergency department utilization in a hospital in the Eastern region of Saudi Arabia. Saudi Medical Journal, 2007, 28:236-240.

2. Scheefer S, Mancuso G, Stein-Mergen M. Augmentation de l'affluence des patients aux urgences du Centre Hospitalier de Luxembourg. Qui sont-ils ? Pourquoi viennentils? [Increase in the inflow of emergency patients at the Central Hospital of Luxembourg. Who are they? Why do they come?]. Bulletin de laSociété des Sciences Médicales du Grand-Duché de Luxembourg, 2005, 1:91-106.

3. Gentile $S$ et al. Non urgent patients in the emergency department? A French formula to prevent misuse. BMC Health Services Research 2010, 10: 66.

4. Chahed MK, Somrani N, Achour H. L'offre et l'utilisation des services d'urgence en Tunisie : principaux resultants d'une enquête nationale exhaustive [Availability and use ofemergency services in Tunisia: main results of a comprehensive national survey]. RSMO 2001. 7 (4/5): 805-811.

5. Comprendre les temps d'attente dans les services d'urgence : Qui utilise les services d'urgence et quels sont les temps d'attente ? [Understanding Emergency Department Wait Times: Whois Using Emergency Departments and How Long Are TheyWaiting?]. Ottawa, Institut canadien d'information sur la santé (ICIS), 2005 (http://secure.cihi.ca/cihiweb/products/Wait_tim es_f.pdf, consulté le 25 octobre 2011)

6. Burt CW, McCaig LF. Trends in hospital emergency department utilization. United States, 1992-99. Vital Health Statistics13 2001. 150:1-34

7. H.L. Ben Gobrane, H. Aounallah - Skhiri, A. Ben Hamida, N. Somrani, M. Ayachi; N. Achour et M. Hsairi. Motifs de recours aux services d'urgence des principaux hôpitaux du Grand Tunis.RSMO 2012 ; 1(18):56-64.

8. Touré P S, Léye A, Pape Mamadou Diop, Léye $Y$ M, Diop M, Ndiaye N, El fjri S, Fall M, Tall C T, Niasse A, Phiri A, Ka M M. Profil épidémio - clinique des patients observés dans un service d'urgences médicales dans un hôpital de banlieue. RAMUR 2012 ; 17 (2) : 3 9 . 
9. K Sall B, Kane O, Diouf E, Bèye MD. Les urgences dans un centre hospitalier et universitaire (CHU) en milieu tropical, le point de vue de l'anesthésiste réanimateur. Med Trop $2002 ; 62: 247-250$.

10. Askénasi R, Gillet J B, Lheureux P. Profil des admissions dans un service d'urgences. Rean Soins Intens Med Urg $1987 ; 3: 201-5$.

11. Rusterholtz T, Pradier C, Potel G, Boyer O, Touze MD, Baron D. Évaluation de l'exactitude diagnostique et du délai thérapeutique dans un service d'urgences médicales. Rean Soins Intens Med Urg $1991 ; 7$ : 199 - 203.

12. Thoër C, Pierret J, Josy Lévy J. Quelques réflexions sur des pratiques d'utilisation des médicaments hors cadre médical. Drogues, santé et société $2008 ; 7$ (1) : 19-54

13. Capewell $\mathrm{S}$. The continuing rise in emergency admissions. British Medical Journal 1996 ; 312 (7037) :991-992.

14. Fourestié V, Roussignol E, Elkarrat $D$, Rauss A, Simon N. Classification clinique des malades aux urgences: définition et reproductibilité. RéanUrg, 1994, 3,573-578.

15. Sagnes-Raffy, Claudet I, Grouteau E, Fries F, Ducassé JL. Epidémiologie des urgences médicales de l'enfant de moins de deux ans. Observatoire Régional des

Urgences de Midi-Pyrénées; 2002.

16. Recensement général de la population et de I'habitat (RGPH) 2009, Mali

17. V. Roger-Leroia, C. Laléchère-Lestradea, S. Tubert-Jeannina.

Caractéristiques des patients ayant recours à l'unité d'urgence odontologique du $\mathrm{CHU}$ de $\begin{array}{cc}\text { Clermont-Ferrand } & \text { (France).Revue } \\ \text { d'Épidémiologie et de Santé Publique } 55 \text { (2007) 197- }\end{array}$ 202.

18. G. Delmas, O. Pierrad, A. Weissenbach, E. Chouvet, D. Schinkel, P. Gillet, R. Streiff, C. Rothmann.Profil des malades admis en salle d'accueil des urgences vitales (SAUV). Journal Européen des urgences. 2007 (1S); 20 : 194 - 195.

19. Abdoulaye Traoré, Hermann Zosé Ouédraogo, Blaise Sondo, Innocent Pierre

Guissou. Les urgences médicales au Centre hospitalier national Yalgado Ouédraogo de Ouagadougou : profil et prise en charge des patients. Cahiers d'études et de recherches francophones/Santé. $2000 ; 12$ (3) : 307-12

20. Berraho M, Boly A, Tachfouti N, Elmajjaoui A, Nejjari C. Les consultations non appropriées aux services des urgences : étude dans un hôpital provincial au Maroc. Prat Organ Soins 2012;43(3):197-204.

21. Rowley ST, Sheller B, Williams BJ, Mancl L. Utilization of a hospital for treatment of pediatric dental emergencies. Pediatr Dent 2006; 28:10-7.

22. Binam F, Takongmo S, Kingue S, Mbanya D, Njip JM, Hagbe P. Circuit de prise en charge des urgences au Cameroun : état des lieux dans un hôpital de la ville de Yaoundé. JEUR 2001; 14 : $233-9$.

23. Mbutiwi IN, Meert $P$, Malengreau $M$, Nseka NM, Dramaix - Wilmet M, Longo LA, Lepira BF. Profil épidémiologique des patients admis dans le service des urgences médicales des Cliniques Universitaires de Kinshasa (République Démocratique du Congo) Ann Afr. Med. Vol 5, № 2, Mars 2012.
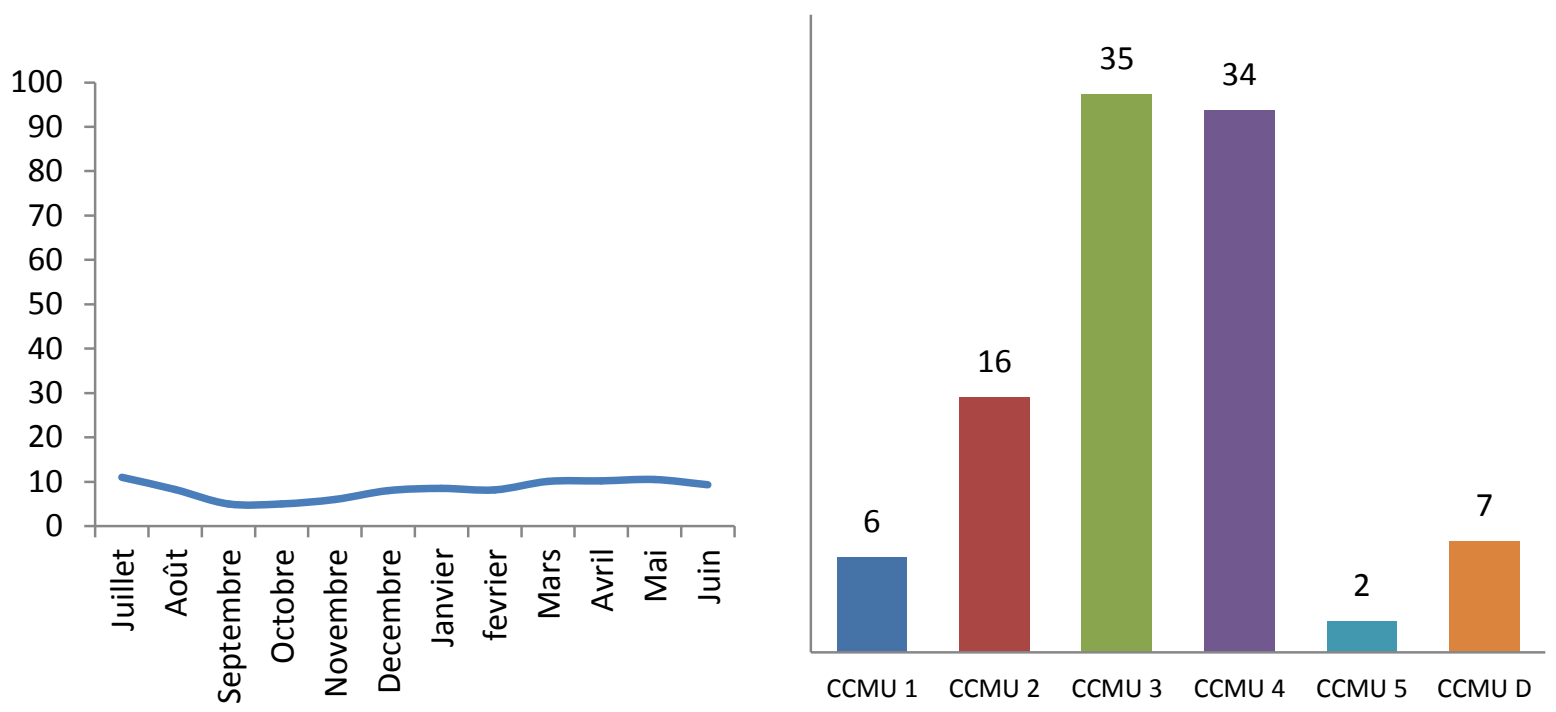

Figure1 : Distribution mensuelle des admissions

Figure2: CCMU: Classification Clinique des Malades des Urgences (modifiée) 
Tableau 2 : Facteurs prédictifs de la gravité de l'état clinique des patients à l'admission

\begin{tabular}{|c|c|c|c|}
\hline Variables & Stable & Grave & $\mathbf{P}$ \\
\hline \multicolumn{4}{|l|}{ Sexe } \\
\hline Masculin & 292 & 917 & \multirow{2}{*}{0,15} \\
\hline Féminin & 180 & 710 & \\
\hline \multicolumn{4}{|l|}{ Tranche d'âge en année } \\
\hline 10 à 20 & 70 & 124 & \multirow{8}{*}{0,001} \\
\hline $21-30$ & 96 & 219 & \\
\hline $31-40$ & 66 & 237 & \\
\hline $41-50$ & 61 & 259 & \\
\hline $51-60$ & 73 & 276 & \\
\hline $61-70$ & 56 & 263 & \\
\hline $71-80$ & 42 & 152 & \\
\hline sup 80 & 8 & 97 & \\
\hline \multicolumn{4}{|l|}{ Niveau d'étude } \\
\hline Pas d'étude & 255 & 986 & \multirow{5}{*}{0.001} \\
\hline Primaire & 80 & 288 & \\
\hline Secondaire & 66 & 229 & \\
\hline Supérieur et Universitaire & 49 & 64 & \\
\hline Technique et Professionnelle & 22 & 60 & \\
\hline \multicolumn{4}{|l|}{ Durée d'évolution de la maladie (jour) } \\
\hline$>7$ & 241 & 747 & \multirow{2}{*}{0,05} \\
\hline$<7$ & 231 & 880 & \\
\hline \multicolumn{4}{|c|}{ Nombre de structures fréquentées avant l'admission } \\
\hline 0 & 93 & 98 & \multirow{3}{*}{0,001} \\
\hline 1 à 3 & 344 & 1433 & \\
\hline$>3$ & 35 & 96 & \\
\hline \multicolumn{4}{|l|}{ Automédication } \\
\hline Oui & 825 & 52,00 & \multirow{2}{*}{0,05} \\
\hline Non & 770 & 48,00 & \\
\hline \multicolumn{4}{|l|}{ Mode de référence } \\
\hline Médicale & 188 & 1081 & \multirow{2}{*}{0.001} \\
\hline Autoréférence & 284 & 546 & \\
\hline \multicolumn{4}{|l|}{ Heure d'admission } \\
\hline Ouvrable & 432 & 1426 & \multirow{2}{*}{0,008} \\
\hline Non ouvrable & 40 & 201 & \\
\hline \multicolumn{4}{|l|}{ Origine de la pathologie } \\
\hline Non infectieuse & 153 & 716 & \multirow{3}{*}{0,004} \\
\hline Infectieuse & 182 & 625 & \\
\hline imprécise & 137 & 686 & \\
\hline
\end{tabular}




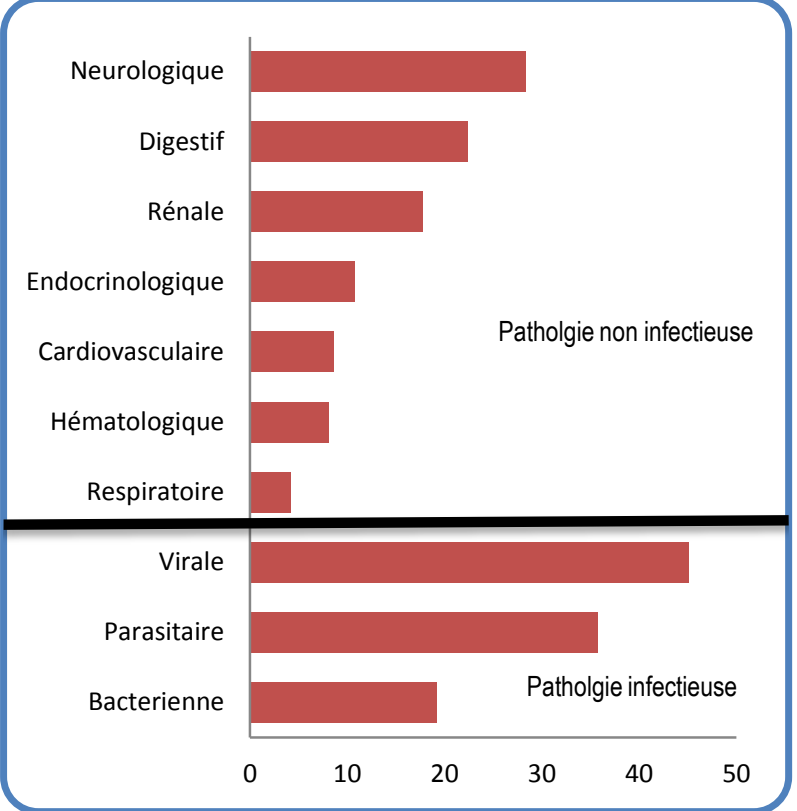

Tableau 1 : Historique de la maladie actuelle

\begin{tabular}{lcc}
\hline & $\mathbf{n}$ (2099) & Pourcentage \\
\hline $\begin{array}{l}\text { Durée d'évolution } \\
\quad \mathbf{7} \text { jours }\end{array}$ & 988 & 47.1 \\
$\quad>7$ jours & 1111 & $\mathbf{5 2 . 9}$ \\
$\begin{array}{l}\text { Nombre de structure } \\
\text { fréquentée avant l'admission }\end{array}$ & & \\
$\quad 0$ & 191 & 31,6 \\
$\quad 1$ à 3 & 1777 & 84.7 \\
$\quad>3$ & 131 & 6.2 \\
$\begin{array}{l}\text { Mode de référence } \\
\quad \text { Médicale }\end{array}$ & 1249 & $\mathbf{5 9 . 5}$ \\
$\quad$ Autoréférence & 850 & 40.5 \\
Automédication & & \\
$\quad$ Oui & 1062 & $\mathbf{5 0 . 6}$ \\
$\quad$ Non & 1037 & 49.4 \\
\hline
\end{tabular}

Figure 3: Distribution des pathologies non infectieuses et infectieuses

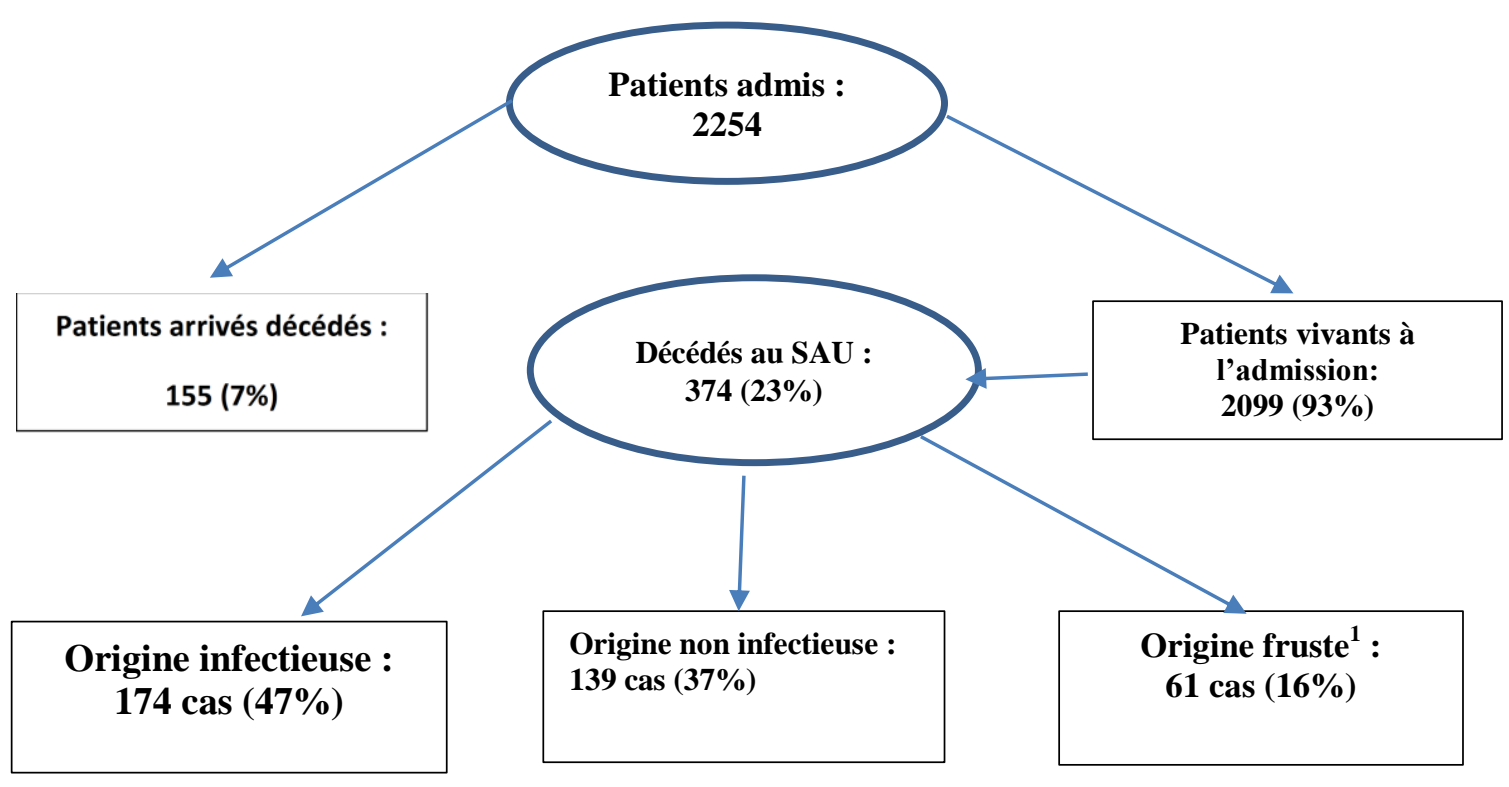

Figure4: Létalité au SAU

1 : Patients chez qui aucune orientation diagnostique claire de l'origine de la pathologie n'a pu être établie 